\title{
Research on the Parameter Identification of LuGre Tire Model Based on Genetic Algorithms
}

\author{
Derong Tan Yanyang Wang Li Zhang \\ School of Automobile and Traffic Engineering, Shandong University of Technology, Zibo 255049, P.R.China
}

\begin{abstract}
The LuGre tire model is a dynamic tire friction model which can describe the dynamic characteristic, but because of its high nonlinearity, it is very difficult to identify the parameters of the model. A two-step method for the parameter identification of LuGre tire model based on genetic algorithms is presented in this paper. In the first step, four static parameters are estimated via PD control, and in the second step, two dynamic parameters are obtained by the PID control. In the two steps, genetic algorithms are used to minimize the identification error.
\end{abstract}

Keywords: PID control, Parameter identification, Genetic algorithms

\section{Introduction}

The problem of predicting the friction force between the tire and the ground for wheeled vehicles is of enormous importance to the automotive industry. Since friction is the major mechanism for generating forces on the vehicle, it is extremely important to have an accurate characterization of the friction force generated at the interface. However, accurate tire/ground friction models are difficult to obtain analytically. Subsequently, in the past several years, the problem of modeling and predicting tire friction has become an area of intense research in the automotive community.

The most common class of tire fiction models in research and applications is the so-called "static" models [1]-[3]. These models predict the friction force for constant vehicle and tire angular velocity as a static map of the relative velocity of the tire to tire friction force. The major representative of this class of models is Pacejka's "Magic Formula" [3]. This is a semiempirical model using a set of parameters to fit a mathematical formula to experimental data. The experimental data in this case correspond to steadystate operation of a tire, which is constant speed, angular rate and slip angle. Such conditions are not reached except during the rather particular case of cruising at constant speed. The development of the friction force at the tire/road interface is very much a dynamic phenomenon. In other words, the friction force does not reach its steady-state value instantaneously, but rather exhibits transient behavior which may differ significantly from its steady-state value. Experiments have shown that the tire/road forces do not necessarily vary along the steady-state force versus slip curves, but rather "jump" from one steady-state curve to the other. In addition, in realistic situations, these variations are most likely to exhibit hysteresis loops, clearly indicating the dynamic nature of friction.

Recently, a second class of tire friction models has been developed that capture the dynamic behavior of friction forces-the so-called "dynamic tire friction models" [4]-[6]. A physics-based dynamic friction model for point contact, the LuGre friction model [5], is applied to the contact patch of a tire and a new tire friction model is developed. The new model provides physical interpretation of the friction forces as a result of elastic deformation of the surfaces in contact. Its dynamic nature allows the study of the transient behavior of tire friction while the singularities associated with slip quantities are avoided.

But it is difficult to identify the parameters of LuGre tire model because of its high nonlinearity. The parameter $Z$ is immeasurable, which describes the state of inner friction. And the coupling fluence between the static parameters and dynamic parameters is serious. In literature [7], Efstatios Velenis proposed a fitting identification method to identify the static parameters by comparing the steady-state characteristics of the model to steady-state data. But the precision is difficult to be ensured because this method will introduce great error when it takes Magic Formula model as fitting standard. Genetic algorithm [8] is a probability searching algorithm about autoadapted global optimization which is formed when simulating the genetic and evolution process of the biology. Comparing to the optimization method based on the gradient and the least squares, genetic algorithms need not the model message of its object when settling nonlinearity problems and these methods can avoid partial minimum. Consequently, 
they have broad adaptability and strong robustness. Genetic algorithms have been applied successfully in the parameter identification of nonlinearity systems. Therefore, a method for the parameter identification of LuGre tire model based on genetic algorithms is presented in this paper.

\section{LuGre tire model}

LuGre tire model is a dynamic tire friction model which is proposed by Canudas de Wit C. It takes the friction phenomenon simulation for the contact face in mutual function between the bristles. Surfaces are very irregular at the microscopic level and two surfaces therefore make contact at a number of asperities. We visualize this as two rigid bodies that make contact through elastic bristles. When a tangential force is applied, the bristles will deflect like springs which give rise to the friction force; see Fig. 1.

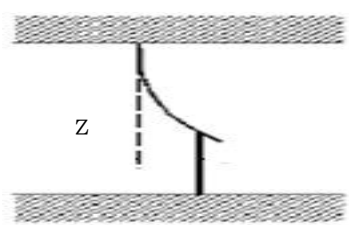

Fig. 1: The friction interface between two surfaces is thought of as a contact between bristles.

If the force is sufficiently large, some of the bristles deflect so much that they will slip. The phenomenon is highly random due to the irregular forms of the surfaces. The LuGre tire friction model bases on the average behavior of the bristles and it is defined by the following set of equations:

$$
\begin{aligned}
& \dot{Z}=v_{r}-\frac{\sigma_{0}\left|v_{r}\right|}{g\left(v_{r}\right)} z \\
& g\left(v_{r}\right)=\mu_{c}+\left(\mu_{s}-\mu_{c}\right) e^{\left(-\left|v_{r} / v_{s}\right|^{1 / 2}\right)} \\
& F=\left(\sigma_{0} z+\sigma_{1} \dot{z}+\sigma_{2} v_{r}\right) F_{n}
\end{aligned}
$$

where $F$ is the normalized friction force, $\sigma_{0}$ the normalized rubber longitudinal lumped stiffness, $\sigma_{1}$ the normalized rubber longitudinal lumped damping, $\sigma_{2}$ the normalized viscous relative damping, $\mu_{\mathrm{s}}$ the normalized static friction, $\mu_{c}$ the normalized Coulomb friction, $v_{s}$ the Stribeck relative velocity, $Z$ the internal weighted mean friction state, $F_{n}$ the total normal force and $v_{r}=r \dot{\theta}-v$ the relative velocity.

In the LuGre tire model, $v$ is one of the important factors which influence the precision of the estimated parameters .However, direct measurement of vehicle velocity such as optical correlation method or spatial filtering method, although available, is often too expensive and requires additional wiring, which makes the system more complex. Relying on an additional sensor also makes the system more prone to sensor failures, thus lowering overall system reliability. Kalman filter and fuzzy logic estimation require at least one more sensor except the wheel velocity sensors. In this paper, we adopt an adaptive nonlinear filter approach to estimate the vehicle velocity based on wheel velocities, which requires only two or four measured wheel velocities. There are no acceleration or yaw rate signals available. In literature [9], the method is studied and tested.

In this paper we consider the simplified motion dynamics of a quarter-vehicle model. The system is then of the form

$$
\begin{aligned}
& m \dot{v}=F \\
& J \ddot{\theta}=-r F+\mu
\end{aligned}
$$

where $m$ is $1 / 4$ of the vehicle mass and $J, r$ are the inertia and radius of the wheel, respectively. $v$ is the linear velocity of the vehicle, $\theta$ is the rolling angle of the wheel, and $\mu$ is the accelerating(or braking) torque. For the sake of simplicity, only longitudinal motion will be considered. The dynamics of the braking and driving actuators are also neglected.

The relationship between the one-wheel model and the LuGre tire friction model is built by formulas (4a), (4b) and (1), (2), (3).

\section{GA-based friction parameter identification}

Static and dynamic parameters identification of LuGre tire model is carried on by two steps: firstly, under the steady state of LuGre tire model, static parameters as $\mu_{s}, \mu_{c}, v_{s}, \sigma_{2}$ are estimated via PD control; then actual value is instead by the gained estimated value of static parameters, dynamic parameters as $\sigma_{0}, \sigma_{1}$ are obtained by the PID control. The simplified motion dynamic of a quarter-vehicle model closed loop system with PD (PID) control is shown in Fig. 2.

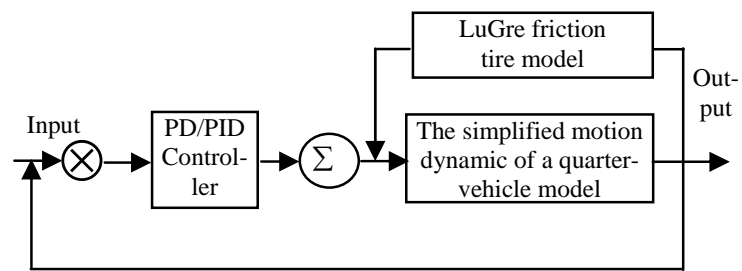

Fig. 2: The simplified motion dynamic of a quarter-vehicle model with a PD/PID controller. 


\subsection{Static parameter identification}

The steady-state characteristics of the LuGre tire friction model are obtained by setting $\dot{Z}=0$, and by imposing that velocities $v$ and $\dot{\theta}$ (and hence $v_{r}$ ) are constant. In this case, the equations of (3) and (1) become

$$
\begin{aligned}
& F_{s}=\left(\sigma_{0} z+\sigma_{2} v_{r}\right) F_{n} \\
& z=\frac{g\left(v_{r}\right)}{\sigma_{0}} \operatorname{sign}\left(v_{r}\right)
\end{aligned}
$$

We can now compute the steady-state expressions for the forces using (5) and (6). In particular, we have

$$
\begin{aligned}
F_{s}= & {\left[\mu_{c}+\left(\mu_{s}-\mu_{c}\right) e^{\left(-\left|(r \dot{\theta}-v) / v_{s}\right|^{1 / 2}\right)}\right] * } \\
& \operatorname{sign}(r \dot{\theta}-v) F_{n}+\sigma_{2}(r \dot{\theta}-v) F_{n}
\end{aligned}
$$

When $\dot{\theta}$ is constant, the (4b) becomes

$$
\mu=r F
$$

Supposing that the closed loop system rotates with the constant speed $\{\dot{\theta}\}_{i=1}^{N}$, and the corresponding control force is $\{\dot{\mu}\}_{i=1}^{N}$. Therefore, $\{\dot{\theta}\}_{i=1}^{N}$ and $\{\dot{\mu}\}_{i=1}^{N}$ can describe the static corresponding relation between the friction force and the angular velocity. Define vector $X_{s}$ as $X_{s}=\left[\begin{array}{llll}\mu_{s} & \mu_{x} & v_{s} & \sigma_{2}\end{array}\right]$. At this point, the identification error is written as

$$
e\left(X_{s}, \dot{\theta}_{i}\right)=\mu_{i}-r F_{s}\left(X_{s}, \dot{\theta}_{i}\right)
$$

where $F_{s}=\left(X_{s}, \dot{\theta}_{i}\right)$ is confirmed by formula (7).

The objective function can be expressed as

$$
J=\sum_{i=1}^{N} e^{2}\left(X_{s}, \dot{\theta}_{i}\right)
$$

Then identification issue is minimizing the objective function $J$.

\subsection{Dynamic parameter identification}

A closed-loop PID control system scheme is adopted in the dynamic parameters identification. The result of static parameters identified in the previous step is used in this step.

The control force $\mu$ in equation (4a) is given by a PID controller

$$
\mu=-k_{p}\left(\theta-\theta_{d}\right)-k_{d} \dot{\theta}-k_{i} \int\left(\theta-\theta_{d}\right) d t
$$

where $k_{p}$ is the proportional constant, $k_{d}$ the differential coefficient constant, and $k_{i}$ the integral constant.

Supposing that the dynamic friction parameter vector is identified as $X_{d}=\left[\begin{array}{ll}\sigma 0 & \sigma 1\end{array}\right]^{T}$, similarly the identification error can also be written as

$$
e\left(X_{d}, t_{i}\right)=\theta\left(t_{i}\right)-\theta_{1}\left(X_{d}, t_{i}\right)
$$

where $\theta\left(t_{i}\right)$ denotes the exact angle of the actual system at time $t_{i} ; \theta_{1}\left(X_{d}, t_{i}\right)$ the exact angle of identified-parameter-embedded system at time $t_{i}$.

Consequently, equations (1), (3) and (4b) become

$$
\begin{aligned}
& J \dot{\theta}=-r F+\mu \\
& F=\left(\sigma_{0} z+\sigma_{1} \dot{z}+\sigma_{2}\left(r \dot{\theta}-v_{1}\right)\right) F_{n} \\
& \dot{z}=\left(r \omega_{1}-v_{1}\right)-\frac{\sigma_{0}\left|r \dot{\theta}_{1}-v_{1}\right|}{g\left(r \dot{\theta}_{1}-v_{1}\right)} z
\end{aligned}
$$

In this case the objective function can be defined as follows

$$
J=c_{1} \sum_{i=1}^{N} e^{2}\left(X_{d}, t_{i}\right)+c_{2} \max \left\{\left|e\left(X_{d}, t_{i}\right)\right|\right\}
$$

where $c_{1}$ and $c_{2}$ are weight coefficients. Then identification issue is minimizing the objective function $J$.

\subsection{Design of genetic algorithms}

In the static and dynamic parameters identification, genetic algorithms [10] are used to minimize the identification error. Parameters which are identified are concatenated together to create a chromosome. Then we encode each parameter using Gray coding. Selection is applied to the current population to create an intermediate population. The individual with the optimal fitness is selected for reproduction. A random choice is made in crossover, where the likelihood of crossover is 0.5. Mutation is applied to each child individually after crossover. It randomly alters each gene with gauss operator. In the process of estimating of dynamic parameters, the probability of mutation adjusts to the number of generations.

The size of population is defined as $M, X_{i}(i=1,2, \ldots M)$ the individual, $f\left(X_{i}\right)$ the fitness function. In the first step, the function to be optimized is the equation (10), and fitness function is given by

$$
\left\{\begin{array}{c}
C_{m}=\max \left\{J\left(X_{i}\right)\right\} \\
f\left(X_{i}\right)=C_{m}-J\left(X_{i}\right)
\end{array} \quad i=1,2, \cdots M\right.
$$

Similarly, the function to be optimized in the second step is the equation (12), and fitness function is as follow

$$
f\left(X_{i}\right)=\frac{1}{J\left(X_{i}\right)} \quad i=1,2, \cdots, M
$$


A block diagram of the estimating method using genetic algorithms is shown in Fig.3.

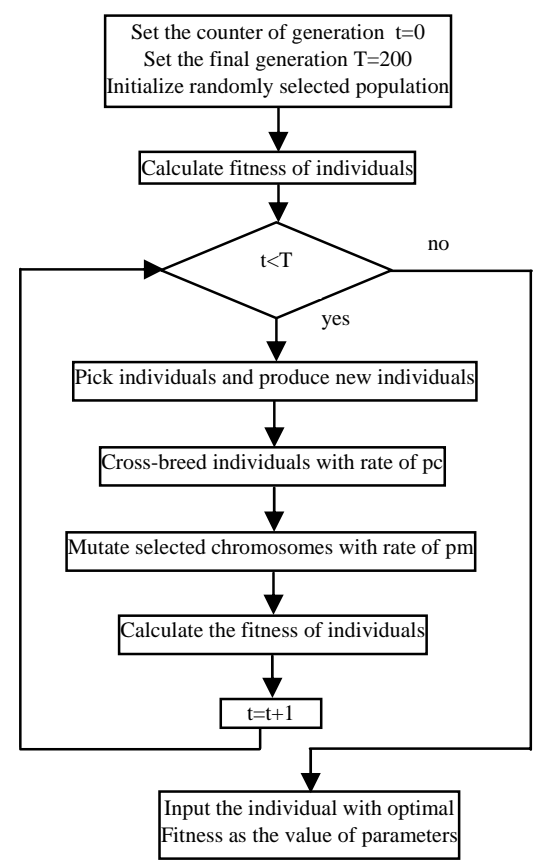

Fig. 3: Flow diagram for the parameters identification using genetic algorithms.

\section{Simulation experiment}

In order to verify the effectiveness of the proposed genetic algorithms in identifying the LuGre tire friction parameters, simulations have been performed with the one-wheel system and the LuGre model. The exact friction parameters used in the simulations are the ones given in Table 1, with the following additional values for the wheel: $r=25 \mathrm{~cm}, m=5 \mathrm{~kg}$, $F_{n}=14 \mathrm{Kgm}^{2} / \mathrm{s}^{2}, J=0.75 * m * r^{2}=0.2344 \mathrm{Kgm}^{2}$. In the process of identifying the static parameters, the initial parameters of PD controller set as follows: $k_{p}=30, k_{d}=0.8$. And the other values are: $T_{1}=200$, $M=50, p c=0.6, p m=0.001$. The search space as follows, $\sigma_{2} \in[0,1], \mu_{c} \in[0,10], \mu_{s} \in[0,10]$, $v_{s} \in[0,50]$.For the static LuGre friction parameters of the system, the offline identified friction parameter values are given in Table 1. Fig.4 shows step responses for system in identifying static parameters. The step responses are quite close. Fig. 5 shows the evolution of the objective function. As we can observe, a good convergence is obtained. Similarly, in the process of identifying the dynamic parameters, the initial parameters of PID controller set as follows: $k_{p}=50, k_{d}=0.5, k_{i}=0.3$. The input is $\theta_{d}(t)=1(t)$. And the other values are: $T_{2}=200, m=50, p c=0.6$, $p m=0.1-(0.1-0.001) * m / M \quad$, where $m$ is currently generation. The search space as follows, $\sigma_{0} \in[0,500], \sigma_{1} \in[0,10]$. The dynamic parameters identified are shown in Table 1 . Step responses are shown in Fig. 6 and the evolution of the objective function are shown in Fig.7.

\section{Conclusions}

A method for identifying the LuGre tire friction parameters using genetic algorithms is proposed in this paper. The shortcomings of great errors and a highly controlled laboratory environment in the fitting method are avoided. Simulation results show that the approaches are effective. In this scheme, wheel velocities signal of anti-lock brake system is only required, and so the expenditure of hardware is small, the realization of procedure is simple. Consequently, the method for the parameter identification of dynamic LuGre tire model presented in this paper has highly engineering practical value.

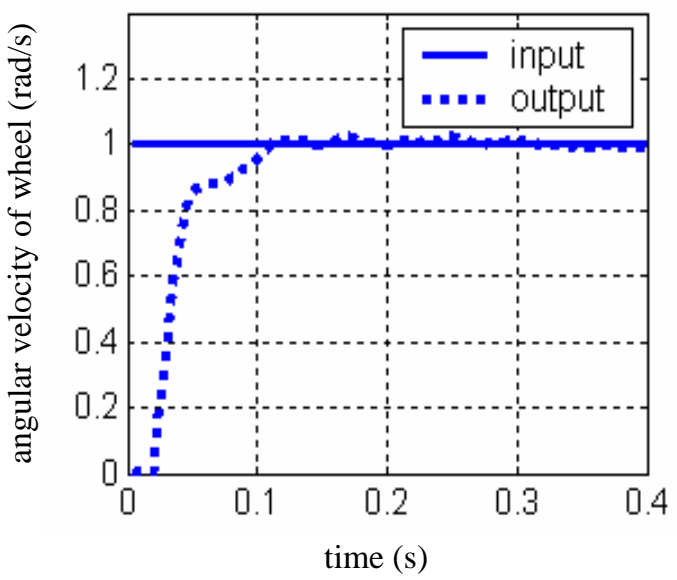

Fig. 4: Step responses of PD controller.

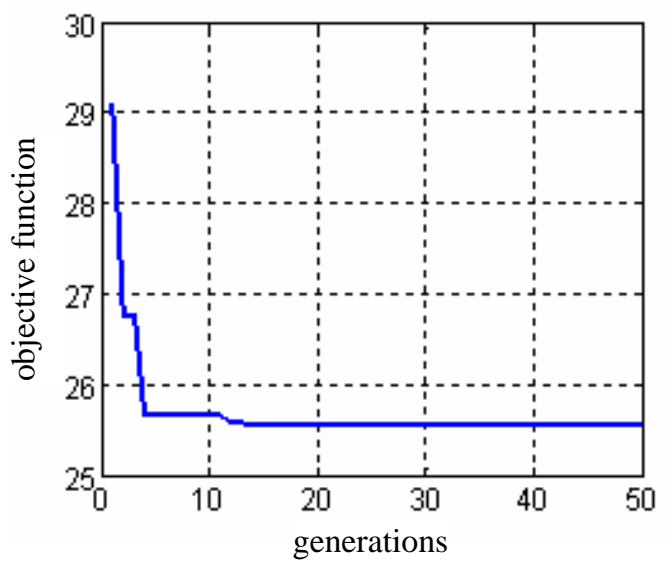

Fig. 5: The evolution of objective function in identifying the static parameters. 


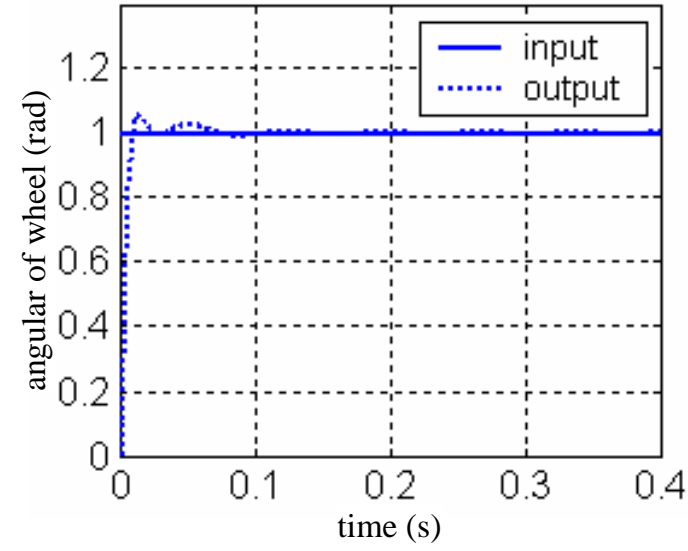

Fig. 6: Step responses of PID controller.

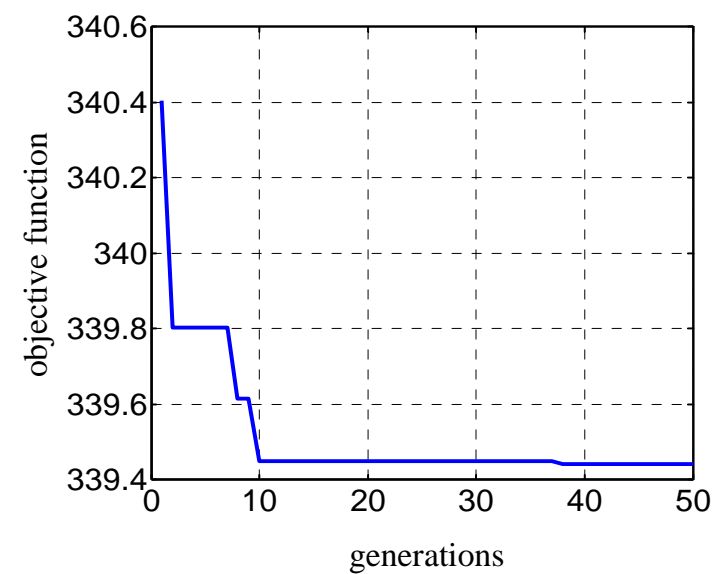

Fig.7: The evolution of objective function in identifying the dynamic parameters.

\begin{tabular}{|l|l|l|}
\hline $\begin{array}{l}\text { Parameter } \\
\text { (unit) }\end{array}$ & True values & Identified value \\
\hline$\sigma_{0}(1 / \mathrm{m})$ & 181 & 183.9803 \\
\hline$\sigma_{1}(\mathrm{~s} / \mathrm{m})$ & 1 & 1.0109 \\
\hline$\sigma_{2}(\mathrm{~s} / \mathrm{m})$ & 0.002 & 0.0019 \\
\hline$\mu_{c}$ & 0.6 & 0.5982 \\
\hline$\mu_{s}$ & 1.5 & 1.5001 \\
\hline$v_{s}(\mathrm{~m} / \mathrm{s})$ & 12.5 & 13.4987 \\
\hline
\end{tabular}

Table 1: Parameter Estimates Vs.True Values.

\section{References}

[1] U. Kiencke and A. Daiss, Estimation of tyre friction for enhanced ABS systems, JSAE Review, 16:221-226,1995.

[2] M. Burckhardt, Fahrwerktecknik: Radschlup fregel system, Vogel-Verlag, 1993.
[3] H. Pacejka and E. Bakker, The magic formula tyre model, Vehicle System Dynamics, 21:13-19, 1993.

[4] C.C.D Wit, M.L. Petersen and A. Shiriaev, A new nonlinear Observer for Tire/Road Distributed Contact Friction, Prof. of the International Conference on Decision and Control, 2003. Proceedings. 42nd IEEE Conference, pp.2246-2251, 2003.

[5] C.C.D. Wit, H. Olsson, K. J. Astrom and P. Lischinsky, A new model for control of systems with friction, Prof. of the International Conference on IEEE Transactions on Automatic Control, pp. 419-425, 1995.

[6] C.C. D Wit, P. Tsiotras and E. Velenis, Dynamic tire friction models for Longitudinal Road/Tire Interation, Vehicle System Dynamics, 12:43-49, 2003.

[7] E. Velenis, Analysis and Control of High-Speed Wheeled Vehicles, Doctor of philosophy, Georgia Institute of Technology, 2006.

[8] K.S. Tang and K. F. Man, Genetic algorithms and their applications, Signal Processing Magazine, 5:54-59, 1996.

[9] J.F. Gao, An adaptive nonlinear filter approach to vehicle velocity estimation for ABS, Prof. of the International Conference on Control Applications, 2000. Proceedings of the 2000 IEEE International , pp.490-495, 2000.

[10] D. Goldberg, On self-adaptive features in realparameter evolutionary algorithms, Evolutionary Computation, 10:23-28, 2001. 